\title{
Open problems on graph eigenvalues studied with AutoGraphiX
}

\author{
Mustapha Aouchiche • Gilles Caporossi • \\ Pierre Hansen
}

Received: 22 May 2012 / Accepted: 19 October 2012 / Published online: 20 November 2012 (C) Springer-Verlag Berlin Heidelberg and EURO - The Association of European Operational Research Societies 2012

\begin{abstract}
Since the late forties of the last century, methods of operations research have been extensively used to solve problems in graph theory, and graph theory has been extensively used to model operations research problems and to solve optimization problems on graphs, e.g., shortest paths and network flow problems. More recently, methods of operations research and artificial intelligence have been used to advance graph theory per se, i.e., to find conjectures on graph theory invariants, to refute such conjectures and in some cases to find automated proofs or ideas of proofs. Among other systems, the AutoGraphiX system was developed since 1997 at GERAD (Montreal) by the present authors. Extensive experiments have been conducted which led to 1,700 conjectures, about 800 of which turned out to be easy and could be proved by the system, and about 600 further ones were proved by hand by us or graph theorists from various countries. Moreover, these results led to many generalizations and further papers. In this paper, we study four theoretical problems related to the eigenvalues of (the adjacency matrix of) a connected graph and to which AutoGraphiX was applied. Three of the problems are related to the maximum value of the irregularity, the maximum spectral spread and the upper bound of Nordhaus-Gaddum type on the index, over the class of connected graphs on $n$ vertices. The fourth problem concerns the maximization of the energy (the sum of the absolute values of the eigenvalues) of a connected graph with fixed numbers of vertices and of cycles. We present a brief survey of the papers on or in connection with these problems, and give some new partial results.
\end{abstract}

\footnotetext{
M. Aouchiche $(\varangle) \cdot$ G. Caporossi · P. Hansen GERAD and HEC Montréal, Montreal, Canada e-mail: mustapha.aouchiche@gerad.ca

G. Caporossi

e-mail: gilles.caporossi@gerad.ca

P. Hansen

e-mail: pierre.hansen@gerad.ca
} 
Keywords Graph $\cdot$ Spectral radius · Irregularity · Spectral spread ·

Nordhaus-Gaddum · Energy

Mathematics Subject Classification (2000) Primary 05C50

\section{Introduction}

In this paper, we consider four open problems related to the eigenvalues of (the adjacency matrix of) a connected graph. The problem is related to the maximum value of the irregularity, i.e., the difference between the spectral radius of a graph and its average degree. Of course, we are also interested in the characterization of the most irregular graphs. The second of these problems concerns the maximum spectral spread, i.e., the maximum value of the difference between the largest and the least eigenvalues of a connected graph, as well as the corresponding extremal graphs. Finding an upper bound of Nordhaus-Gaddum type on the index over the class of connected graphs on $n$ vertices, our third problem, was independently posed by Nosal (1970) and by Amin and Hakimi (1972). More than 40 years later, the problem remains open despite a series of improvements (see Aouchiche and Hansen 2012 for a survey) of the first bound given in Amin and Hakimi (1972) and Nosal (1970). The fourth problem concerns the maximization of the energy (the sum of the absolute values of the eigenvalues), as defined by Gutman $(1978,2001)$, of a connected graph with fixed numbers of vertices and of cycles.

Even if these problems have been studied before, all related conjectures stated in the present paper were accurately formulated (see Aouchiche et al. 2008a) after some experiments with the programming system AutoGraphiX, designed for making conjectures in graph theory. Thus, we first give a brief overview of the use of the computer in general and the AutoGraphiX system in particular, in graph theory.

Computers have been extensively used in graph theory and its applications to various fields since the fifties of the last century. The main use was computation of the values of graph invariants, i.e., quantities such as the independence and chromatic numbers, the radius or the diameter of a graph, which do not depend on the labelling of its vertices or edges. In addition to such tasks of intelligent number-crunching (which imply the design of exact algorithms or heuristics as well as their efficient implementation with well-chosen data-structures Knuth 1993; Mehlhorn and Nähger 1995; Pisanski and Zitnik 2005; Skiena 1990), computers can also be used for graph drawing (di Battista et al. 1994, 1999) and for advancing the theory itself, i.e., finding in a computerassisted or sometimes fully automated way conjectures, proofs and refutations. See Hansen (2005) for a survey and discussion of systems designed for that purpose, as well as the book Graphs and Discovery (Fajtlowicz et al. 2005), in particular chapters 4, 5, 6, 11 and 12 which discuss such systems.

The AutoGraphiX (AGX) system was developed at GERAD, Montreal since 1997. It addresses the following problems:

(i) Find a graph $G$ satisfying given constraints;

(ii) Find a graph $G$ maximizing or minimizing a given invariant, possibly subject to constraints; 
(iii) Find a conjecture, which may be algebraic, i.e., a relation between graph invariants, or structural, i.e., a characterization of extremal graphs for some invariant;

(iv) Corroborate, refute and/or strengthen or repair a conjecture;

(v) Suggest ideas of proof.

The AGX system was described in Aouchiche (2006), Aouchiche et al. (2005), Caporossi (2000), and Caporossi and Hansen (2000); three ways it uses to fully automate conjecture making are presented in Caporossi (2000) and Caporossi and Hansen (2004). Applications to graph theory are given in Aouchiche et al. (2001, 2006, 2007a,b, 2008a,b, 2009a,b,c, 2010, 2011), Aouchiche and Hansen (2005, 2007a,b, 2009, 2011), Belhaiza et al. (2005), Cvetković et al. (2001), Hansen and Mélot (2005), Sedlar et al. (2008), and Stevanović et al. (2008); applications to chemical graph theory in Aouchiche and Hansen (2010), Caporossi et al. (1999a,b), Fowler et al. (2001), Gutman et al. (2005), Hansen and Mélot (2003), Hansen et al. (2005); and developments of these results in Caporossi et al. (2003) and Gutman et al. (1999).

AGX was enriched in various ways over the years. A main goal was to follow closely the way a graph-theorist proceeds with his work, both in the discovery of new conjectures and in the proof or refutation of them. As explained below, this makes various tasks much more efficient and points the way towards obtaining first successes in other ones such as automated proof in graph theory.

The main ideas behind AGX are that

(i) Most problems of extremal graph theory can be viewed as problems of parametric combinatorial optimization of the form

$$
\min / \max _{G \in \mathcal{G}_{n}} i(G) \quad \text { or } \quad \min / \max _{G \in \mathcal{G}_{n, m}} i(G)
$$

for some invariant $i(G)$ with parameters $n$ and $m$, where $\mathcal{G}_{n}$ and $\mathcal{G}_{n, m}$ denote respectively the class of graphs on $n$ vertices and that on $n$ vertices and $m$ edges, or the exploitation of their solutions;

(ii) All problems of the form (1) can be solved approximately by a generic heuristic.

To obtain such a heuristic, the Variable Neighborhood Search metaheuristic (VNS) (Hansen and Mladenović 2001; Mladenović and Hansen 1997), a general framework for building heuristics, is specialized. VNS exploits systematically changes in neighborhoods used in the search, both in a descent phase to obtain a locally extremal graph, and in a "shaking" phase, to get out of the corresponding valley (or away from the corresponding mountain) in order to find a better graph. Neighborhoods correspond to changes brought to the graph $G$, e.g. all ways to add, or to remove an edge, and so forth.

In this paper, we consider only simple, undirected and finite graphs, i.e., undirected graphs on a finite number of vertices without multiple edges or loops. A graph is (usually) denoted by $G=(V, E)$, where $V$ is its vertex set and $E$ its edge set. The order of $G$ is the number $n=|V|$ of its vertices and its size is the number $m=|E|$ of its edges. The degree of a vertex $v \in V$, denoted by $d(v)=d_{G}(v)$ is the number of edges incident to $v$. The average degree in a graph $G$ is denoted by $\bar{d}=\bar{d}(G)$. The complement $\bar{G}$ of $G$ is the graph on the same vertex set as $G$ and in which an edge joins vertices $u$ and $v$ if and only if there is no such edge in $G$. 


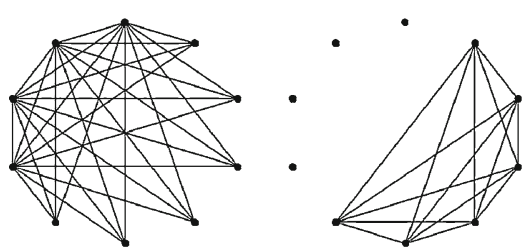

(a)

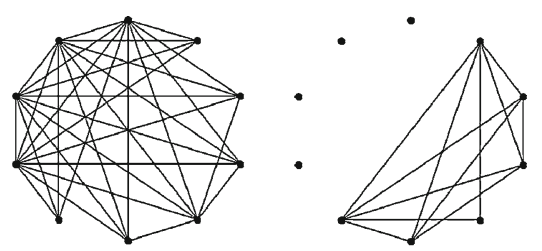

(b)

Fig. 1 a The complete split graph $\mathrm{CS}(10,4)$ and its complement. b The fanned complete split graph $\operatorname{FCS}(10,4,3)$ and its complement
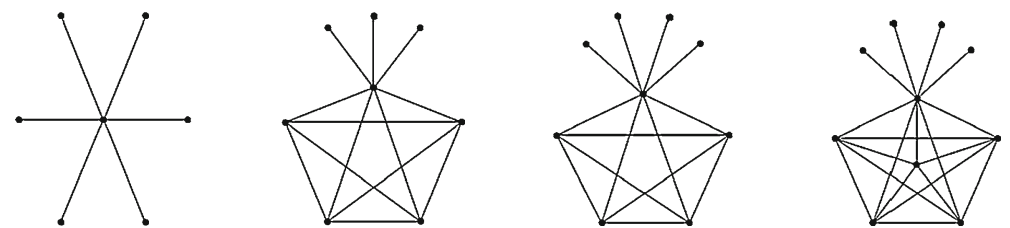

Fig. 2 Presumably most irregular graphs for $n=7,8,9,10$

The adjacency matrix $A$ of $G$ is a $0-1 n \times n$-matrix indexed by the vertices of $G$ and defined by $a_{i j}=1$ if and only if $i j \in E$. Denoted by $\left(\lambda_{1}, \lambda_{2}, \ldots, \lambda_{n}\right)$, the $A$-spectrum of $G$, i.e., the spectrum of the adjacency matrix of $G$, and assume that the eigenvalues are labeled such that $\lambda_{1} \geq \lambda_{2} \geq \cdots \geq \lambda_{n}$.

As usual, we denote by $P_{n}$ the path, by $C_{n}$ the cycle, by $S_{n}$ the star, by $K_{a, n-a}$ the complete bipartite graph and by $K_{n}$ the complete graph, each on $n$ vertices.

A complete split graph with parameters $n, q(q \leq n)$, denoted by $\operatorname{CS}(n, q)$, is a graph on $n$ vertices consisting of a clique on $q$ vertices and an independent set on the remaining $n-q$ vertices in which each vertex of the clique is adjacent to each vertex of the independent set. An example of a complete split graph is given in Fig. 1a.

A fanned complete split graph with parameters $n, q, t(n \geq q \geq t)$, denoted by $\operatorname{FCS}(n, q, t)$, is a graph (on $n$ vertices) obtained from a complete split graph $\operatorname{CS}(n, q)$ by connecting a vertex from the independent set by edges to $t$ other vertices of the independent set. An example of a fanned complete split graph is given in Fig. $1 \mathrm{~b}$.

A pineapple with parameters $n, q(q \leq n)$, denoted by $\mathrm{PA}(n, q)$, is a graph on $n$ vertices consisting of a clique (a set of pairwise adjacent vertices) on $q$ vertices and an independent set (a set of pairwise non-adjacent vertices) on the remaining $n-q$ vertices in which each vertex of the independent set is adjacent to a unique and the same vertex of the clique. Some pineapples are illustrated in Fig. 2.

The remainder of this paper is organized as follows. The next section discusses the problem related to the irregularity of a graph. We give new partial results about the upper bound. In "The spectral spread", after a brief survey of the results related to the problem of upper bounding of the spectral spread of a graph, we compute the spectrum of the extremal graphs. Then, we reformulate the corresponding conjecture in an accurate statement. Section "Nordhaus-Gaddum inequality for the spectral radius" is a survey of results about an upper bound of the Nordhaus-Gaddum type for the index, i.e., an upper bound on $\lambda_{1}(G)+\lambda_{1}(\bar{G})$ where $G$ is a connected graph and $\bar{G}$ its complement. The problem of maximizing the energy of a graph, is discussed in 
"Energy of a graph". We recall known results in the cases of unicyclic and bicyclic connected graphs. We conjecture about the upper bound of the energy in the cases of tricyclic and quadricyclic connected graphs. Finally, we state a conjecture for the general case of $r$-cyclic connected graphs on a fixed number $n$ vertices for a given integer $r$ with $6 r+4 \leq n$.

\section{The irregularity}

Denote by $\lambda_{1}=\lambda_{1}(G)$ the largest eigenvalue, also known as the index or spectral radius, (of the adjacency matrix) of a graph $G$, and by $\bar{d}=\bar{d}(G)$ its average degree. The well-known result, in spectral graph theory, $\lambda_{1}(G) \geq \bar{d}(G)$ with equality if and only if $G$ is a regular graph, was proved by Collatz and Sinogowitz (1957). Then, they proposed to consider the difference between the index and the average degree as a measure of the irregularity of a graph [other definitions of irregularity in graphs have been proposed, see Alberston (1997) and Bell (1992), and for a comparison between them see Gutman et al. (2005)]. Thus, the irregularity of a graph $G$ is defined by $\operatorname{Irr}(G)=\lambda_{1}(G)-\bar{d}(G)$. The problem of finding an upper bound on the irregularity and characterizing the most irregular graphs remains open. The following conjecture related to the irregularity of a graph have been formulated after some experiments with the system AGX.

Conjecture 2.1 The most irregular connected graph on $n(n \geq 10)$ vertices is a pineapple $\operatorname{PA}(n, q)$ in which the clique size $q$ is equal to $\left\lceil\frac{n}{2}\right\rceil+1$.

Extremal graphs have been obtained by AGX and recognized to be pineapple graphs for $n=10,11, \ldots, 20$. For smaller values of $n$ the maximal graph is again a pineapple (reduced to a star for $n=5,6,7)$. For $n \in\{7,8,9,10\}$, the extremal graphs obtained by AGX are illustrated in Fig. 2. The size of the clique was found using AGX's relation finding routine.

Using a result of Hong (1988), $\lambda_{1} \leq \sqrt{m-n+1}$, it is shown in Aouchiche et al. (2008a) and Bell (1992) that

$$
\lambda_{1}-\bar{d} \leq \frac{n}{4}-1+\frac{1}{n}
$$

The values of this bound is very close to what implied by Conjecture 2.1. Actually, $\frac{n}{4}-1$ is the best-fitting linear function for irregularity of pineapples in which the clique size is equal to $\left\lceil\frac{n}{2}\right\rceil+1$ : its index is slightly larger than $\left\lceil\frac{n}{2}\right\rceil$ (the index is a root of a cubic equation and a nice closed-form formula seems to be out of reach) and its average degree is approximately $\frac{n}{4}+\frac{3}{2}-\frac{2}{n}$. In fact, we can give a lower bound on the maximum value of $\operatorname{Irr}=\lambda_{1}-\bar{d}$.

Lemma 2.2 For an integer $n \geq 10$, let $b_{n}=\max \{\operatorname{Irr}(G): G$ connected and $|G|=n\}$. Then

$$
b_{n}> \begin{cases}\frac{n}{4}-\frac{3}{2}+\frac{2}{n} & \text { if } n \text { is even } ; \\ \frac{n}{4}-\frac{3}{2}+\frac{7}{4 n} & \text { if } n \text { is odd. }\end{cases}
$$


Proof It is trivial that $b_{n} \geq \lambda_{1}(\mathrm{PA}(n, q))-\bar{d}(\operatorname{PA}(n, q))$, for any pineapple $\mathrm{PA}(n, q)$. Thus, it suffices to prove the inequality for a pineapple $\operatorname{PA}(n, q)$ with $q=\left\lceil\frac{n}{2}\right\rceil+1$. We have, on one hand

$$
\bar{d}(\mathrm{PA}(n, q))=\frac{q(q-1)+2 n-2 q}{n}=\frac{\left\lceil\frac{n}{2}\right\rceil\left(\left\lceil\frac{n}{2}\right\rceil+1\right)}{n}+\frac{2\left\lfloor\frac{n}{2}\right\rfloor-2}{n},
$$

and on the other hand, since $\operatorname{PA}(n, q)$ contains a clique $K_{q}$,

$$
\lambda_{1}(\operatorname{PA}(n, q))>q-1=\left\lceil\frac{n}{2}\right\rceil .
$$

Now, easy computations lead to

$$
\lambda_{1}(\operatorname{PA}(n, q))-\bar{d}(\operatorname{PA}(n, q))> \begin{cases}\frac{n}{4}-\frac{3}{2}+\frac{2}{n} & \text { if } n \text { is even; } \\ \frac{n}{4}-\frac{3}{2}+\frac{7}{4 n} & \text { if } n \text { is odd }\end{cases}
$$

and the result follows.

Conjecture 2.1 is true if the minimum degree of $G$ is assumed to be at least 2, i.e., $\delta(G) \geq 2$. To prove that fact, we need recalling an inequality due to Hong et al. (2001).

Lemma 2.3 (Hong et al. 2001) Let $G$ be a graph on $n$ vertices and $m$ edges with minimum degree $\delta$. Then

$$
\lambda_{1}(G) \leq \frac{\delta-1+\sqrt{(\delta+1)^{2}+4(2 m-\delta n)}}{2}
$$

with equality if and only if $G$ is regular or biregular in which each vertex is of degree either $\delta$ or $n-1$.

Theorem 2.4 Let $G$ be a connected graph on $n$ vertices with minimum degree $\delta \geq 2$. Then

$$
\lambda_{1}(G)-\bar{d}(G) \leq \frac{n}{4}-\frac{3}{2}+\frac{9}{4 n}<\lambda_{1}(\operatorname{PA}(n, q))-\bar{d}(\operatorname{PA}(n, q)),
$$

where $q=\left\lceil\frac{n}{2}\right\rceil+1$.

Proof Let $G$ be a connected graph on $n$ vertices with minimum degree $\delta \geq 2$. Using Lemma 2.3, we have

$$
\begin{aligned}
& \lambda_{1}(G) \leq \frac{\delta-1}{2}+\frac{\sqrt{(\delta+1)^{2}+4(2 m-\delta n)}}{2}=\frac{\delta-1}{2} \\
& +2 \sqrt{\frac{n}{4} \cdot\left(\frac{2 m}{n}+\frac{(\delta+1)^{2}}{4 n}-\delta\right)} \leq \frac{n}{4}+\frac{2 m}{n}+\frac{(\delta+1)^{2}}{4 n}-\frac{\delta+1}{2} .
\end{aligned}
$$


Then

$$
\lambda_{1}(G)-\bar{d}(G) \leq \frac{n}{4}+\frac{(\delta+1)^{2}}{4 n}-\frac{\delta+1}{2},
$$

which bound is decreasing with respect to $\delta$. Therefore, the maximum is reached for $\delta=2$, and

$$
\lambda_{1}(G)-\bar{d}(G) \leq \frac{n}{4}+\frac{9}{4 n}-\frac{3}{2} .
$$

Let

$$
t_{n}=\bar{d}(\mathrm{PA}(n, q))+\frac{n}{4}+\frac{9}{4 n}-\frac{3}{2} .
$$

To be done, it remains to prove that

$$
\lambda_{1}(\operatorname{PA}(n, q))>t_{n}
$$

Consider the matrix $N$ associated to the divisor (cf. Chapter 4 of Cvetković et al. 1995) of a pineapple graph $\operatorname{PA}(n, q)$

$$
N=\left[\begin{array}{ccc}
q-2 & 1 & 0 \\
q-1 & 0 & n-q \\
0 & 1 & 0
\end{array}\right] .
$$

The characteristic polynomial of $N$ is

$$
P_{N}(t)=\operatorname{det}(t I-N)=t^{3}-(q-2) t^{2}-(n-1) t+(n-q)(q-2) .
$$

To compute $P_{N}(t)$, as well as the following expressions, we used the software Maple (A computer algebra system. http://www.maplesoft.com).

Now, we consider two cases according to the parity of $n$. First, if $n$ is even, $q=$ $\lceil n / 2\rceil+1=(n+2) / 2$ and we have

$$
P_{N}\left(t_{n}\right)=-\frac{7}{16} n+1+\frac{5}{16 n}+\frac{1}{16 n^{2}}<0, \quad \text { for all } n \geq 3,
$$

and

$$
P_{N}\left(t_{n}+1\right)=\frac{1}{4} n^{2}+\frac{1}{2} n+1+\frac{9}{2}+\frac{25}{16 n}+\frac{1}{4 n^{2}}>0, \quad \text { for all } n .
$$

Thus, the characteristic polynomial $P_{N}(t)$ has a root between $t_{n}$ and $t_{n}+1$. Finally, if $n$ is odd, $q=\lceil n / 2\rceil+1=(n+3) / 2$ and we have 


$$
P_{N}\left(t_{n}\right)=-\frac{n}{2}+\frac{3}{2}<0, \quad \text { for all } n \geq 4,
$$

and

$$
P_{N}\left(t_{n}+1\right)=\frac{n^{2}+4 n+27}{4}>0, \text { for all } n .
$$

Thus, the characteristic polynomial $P_{N}(t)$ has a root between $t_{n}$ and $t_{n}+1$. Therefore, in both cases $\lambda_{1}(\mathrm{PA}(n, q))>t_{n}$. This completes the proof.

\section{The spectral spread}

Let $\lambda_{n}=\lambda_{n}(G)$ denote the least eigenvalue of a graph $G$. The spectral spread of $G$ is defined by $s(G)=\lambda_{1}(G)-\lambda_{n}(G)$. The problem of finding the maximum value of $s(G)$ among the class of connected graphs of given order $n$ is an open problem. Experiments done with the AutoGraphiX system led to the following conjecture.

Conjecture 3.1 Given n, the maximal value of the spectral spread of a graph on $n$ vertices is obtained for a complete split graph $\operatorname{CS}(n, q)$ with an independent set of size $n-q=\left\lceil\frac{n}{3}\right\rceil$.

Again extremal graphs were found by AGX and the size of the independent set obtained using its relation finding routine.

Little can be found in the literature concerning the spectral spread of a graph. All graphs whose spectral spread does not exceed 4 are determined in Petrović (1983). The spectral spread of unicyclic graphs has been studied in Shu and Wu (2003). Note that Conjecture 3.1 did appear in Gregory et al. (2001), where it has been verified by computer for graphs up to 9 vertices, but remained unsolved. The conjecture is only about the structure of the extremal graphs. To get the corresponding bound on $s(G)$, it is necessary to compute the largest and the smallest eigenvalue of a complete split graph $\operatorname{CS}(n, q)$.

Consider the matrix $M$ associated to the divisor (cf. Chapter 4 of Cvetković et al. 1995) of a complete split graph $\operatorname{CS}(n, q)$

$$
M=\left[\begin{array}{cc}
q-1 & n-q \\
q & 0
\end{array}\right] \text {. }
$$

The eigenvalues of $M$ are

$a_{1}=\frac{q-1}{2}+\frac{\sqrt{4 q n-3 q^{2}-2 q+1}}{2}$ and $a_{2}=\frac{q-1}{2}-\frac{\sqrt{4 q n-3 q^{2}-2 q+1}}{2}$.

The values $a_{1}$ and $a_{2}$ are also the main eigenvalues of $\operatorname{CS}(n, q)$. The index of the graph is exactly the largest eigenvalue of $M$, i.e. $\lambda_{1}(\operatorname{CS}(n, q))=a_{1}$. We will show that $\lambda_{n}(\mathrm{CS}(n, q))=a_{2}$. First, we prove the following result. 
Proposition 3.2 Let $G$ be a connected graph on $n \geq 3$ vertices with maximum degree $\Delta=n-1$ and where at least two vertices have degree $\Delta$. Then

- -1 is an eigenvalue of $G$ with multiplicity at least $n_{\Delta}-1$, where $n_{\Delta}$ is the number of dominating vertices in $G$;

- 0 is an eigenvalue of $G$ with multiplicity at least $\alpha-1$, where $\alpha$ is the independence number of $G$;

- -1 and 0 are not among the main eigenvalues of $G$.

Proof Assume, without loss of generality, that the vertices of $G$ are indexed $v_{1}, v_{2}, \ldots v_{n}$ such that $v_{1}, v_{2} \ldots v_{n_{\Delta}}$ are the dominating vertices, and $v_{n-\alpha}, v_{n-\alpha+1}$, $\ldots v_{n}$ are the vertices of a maximum independent set. Let $A$ denote the adjacency matrix of $G$ and $I$ the identity matrix of order $n$.

- The result is true if the graph is complete. Therefore, assume that $G ¥ K_{n}$. From the definition of an eigenvalue, any vector $X=\left[x_{1}, x_{2}, \ldots, x_{n}\right]^{t}$ is an eigenvector of $G$ belonging to -1 if and only if $(A+I) X=0$. Now, an $n$ vector of the form $X=\left[x_{1}, x_{2}, \ldots, x_{n_{\Delta}}, 0, \ldots 0\right]^{t}$ is an eigenvector of $G$ belonging to -1 if and only if $x_{1}+x_{2}+\cdots+x_{n_{\Delta}}=0$. Thus, -1 is an eigenvalue of $G$ and the corresponding eigenspace has dimension at least $n_{\Delta}-1$.

- As for -1 , an $n$ vector of the form $X=\left[0, \ldots 0, x_{n-\alpha}, x_{n-\alpha+1}, \ldots x_{n}\right]^{t}$ is an eigenvector of $G$ belonging to 0 if and only if $x_{n-\alpha}+x_{n-\alpha+1}+\cdots+x_{n}=0$. Thus, 0 is an eigenvalue of $G$ and the corresponding eigenspace has dimension at least $\alpha-1$.

- It is easy to see that $x_{1}+x_{2}+\cdots+x_{n}=0$ for any of the above eigenvectors, thus -1 and 0 are not among the main eigenvalues of $G$.

Using the above conjecture and the divisor of a complete split graph, we can derive the following result.

Corollary 3.3 The spectrum of a complete split graph $\mathrm{CS}(n, q)$ is

$$
\left(\begin{array}{cccc}
\frac{q-1}{2}+\frac{\sqrt{4 q n-3 q^{2}-2 q+1}}{2} & 0 & -1 & \frac{q-1}{2}-\frac{\sqrt{4 q n-3 q^{2}-2 q+1}}{2} \\
1 & n-q-1 & q-1 & 1
\end{array}\right) .
$$

Now, we reformulate Conjecture 3.1 in order to provide an upper bound in addition to extremal graphs.

Conjecture 3.1' Let $G$ be a connected graph on $n \geq 3$ vertices. Then $s(G) \leq$ $\sqrt{4 q n-3 q^{2}-2 q+1}$ with equality if and only if $G$ is the complete split graph $\mathrm{CS}(n, q)$ with an independent set of size $n-q=\left\lceil\frac{n}{3}\right\rceil$ and a clique of size $q=\left\lfloor\frac{2 n}{3}\right\rfloor$.

\section{Nordhaus-Gaddum inequality for the spectral radius}

Nordhaus and Gaddum (1956) proved that

$$
2 \sqrt{n} \leq \chi(G)+\chi(\bar{G}) \leq n+1 \quad \text { and } \quad n \leq \chi(G) \cdot \chi(\bar{G}) \leq \frac{(n+1)^{2}}{4}
$$


where $\chi$ is the chromatic number of a graph. Finck (1968) showed that these bounds were sharp (taking floors and ceilings if necessary) and characterized extremal graphs. Similar bounds were obtained for a large number of graph invariants by a variety of authors. Let $i(G)$ denote a graph invariant. Classical Nordhaus-Gaddum relations are of the following form:

$$
l_{1}(n) \leq i(G)+i(\bar{G}) \leq u_{1}(n) \quad \text { and } \quad l_{2}(n) \leq i(G) \cdot i(\bar{G}) \leq u_{2}(n)
$$

In more general form, the lower and upper bounding functions may depend on several variables. For an exhaustive survey of such relations see Aouchiche and Hansen (2012) and the references therein. In the present paper, we are interested in NordhausGaddum relations only for the index. Nosal (1970) and Amin and Hakimi (1972) independently proved that

$$
n-1 \leq \lambda_{1}(G)+\lambda_{1}(\bar{G}) \leq \sqrt{2}(n-1) .
$$

The lower bound (attained if and only if the graph is regular) has been proved independently by Nosal (1970) and Amin and Hakimi (1972), and has been improved by Nikiforov (2007) to

$$
\lambda_{1}(G)+\lambda_{1}(\bar{G}) \geq n-1+\sqrt{2} \frac{d i v^{2}(G)}{n^{3}},
$$

where $\operatorname{div}(G)=\sum_{u \in V(G)}\left|d(u)-\frac{2 m}{n}\right|$.

The upper bound attracted more attention. We list some of these upper bounds.

$$
\begin{aligned}
& \operatorname{Li}(1996) \quad \lambda_{1}(G)+\lambda_{1}(\bar{G}) \leq \sqrt{2 n(n-1)-4 \delta(n-1-\Delta)+1}-1 \text {; } \\
& \text { Shi (2007) } \quad \lambda_{1}(G)+\lambda_{1}(\bar{G}) \leq \sqrt{2\left((n-1)^{2}-2 \delta n+2 \Delta \delta-\Delta+3 \delta\right)} \text {; } \\
& \text { Shi (2007) } \quad \lambda_{1}(G)+\lambda_{1}(\bar{G}) \\
& \leq \frac{n-\Delta+\delta-3+\sqrt{2\left((n-\Delta)^{2}+4 n(\Delta-\delta)+(\delta+1)^{2}\right)}}{2} \text {; } \\
& \text { Hong and Shu (2000) } \quad \lambda_{1}(G)+\lambda_{1}(\bar{G}) \leq \sqrt{\left(2-\frac{1}{\chi(G)}-\frac{1}{\chi(\bar{G})}\right) n(n-1)} \text {; } \\
& \text { Nikiforov (2002) } \quad \lambda_{1}(G)+\lambda_{1}(\bar{G}) \leq \sqrt{\left(2-\frac{1}{\theta(G)}-\frac{1}{\theta(\bar{G})}\right) n(n-1)} \text {; }
\end{aligned}
$$

where $\delta, \Delta, \chi$ and $\theta$ denote the minimum degree, maximum degree, chromatic number and maximum clique number, respectively.

The best bound known up to now is proved by Csikvári (2009):

$$
\lambda_{1}(G)+\lambda_{1}(\bar{G}) \leq \frac{1+\sqrt{3}}{2} n-1 .
$$


The AutoGraphiX conjecture about the upper bound is as follows.

Conjecture 4.1 (Aouchiche et al. 2008a; Aouchiche and Hansen 2012) For any simple graph $G$, with complement $\bar{G}$, index $\lambda_{1}(G)$ and $n$ vertices we have

$$
\lambda_{1}(G)+\lambda_{1}(\bar{G}) \leq \frac{4}{3} n-\frac{5}{3}- \begin{cases}f_{1}(n) & \text { if } n \bmod (3)=1 \\ 0 & \text { if } n \bmod (3)=2 \\ f_{2}(n) & \text { if } n \bmod (3)=0\end{cases}
$$

where $f_{1}(n)=\frac{3 n-2-\sqrt{9 n^{2}-12 n+12}}{6}$ and $f_{2}(n)=\frac{3 n-1-\sqrt{9 n^{2}-6 n+9}}{6}$.

This bound is sharp and attained if and only if $G$ or $\bar{G}$ is a complete split graph with an independent set on $\left\lfloor\frac{n}{3}\right\rfloor$ vertices (and also on $\left\lceil\frac{n}{3}\right\rceil$ vertices if $n \bmod (3)=2$ ).

We shall describe in some detail the use of AGX in formulating Conjecture 4.1.

Additional experiments have shown that maximal graphs for $\lambda_{1}+\bar{\lambda}_{1}$ for given $n$ and $m$ are complete split graphs or fanned complete split graphs with a few exceptions.

When looking for extremal graphs with the system AGX, using the variable neighborhood search metaheuristic, we defined the objective function as $\lambda_{1}(G)+\lambda_{1}(\bar{G})$ to be maximized over the class of all graphs of order from 4 to 24 . To be coherent in our investigations, we required the graph $G$, but not necessarily its complement $\bar{G}$, to be connected. This constraint is without loss of generality because of the fact that at least one of the complementary graphs $G$ and $\bar{G}$ is connected.

For a fixed order $n$, the extremal graph $G$ is composed of a clique on $q$ vertices and an independent set with $s$ vertices in which every vertex is connected to all vertices of the clique. When we observed the values of $q$ and $s$ for different graphs, we found the following:

$$
q=\left\{\begin{array}{cl}
\left\lfloor\frac{n}{3}\right\rfloor & \text { if } n \bmod (3)=1 \\
\frac{n}{3} & \text { if } n \bmod (3)=0
\end{array} \quad \text { and } \quad s=\left\{\begin{array}{cc}
\left\lceil\frac{2 n}{3}\right\rceil \text { if } n \bmod (3)=1 \\
\frac{2 n}{3} \text { if } n \bmod (3)=0
\end{array}\right.\right.
$$

While the experiments show regularity for the cases $n \bmod (3)=0$ and $n \bmod (3)=$ 1 , it was not the case when $n \bmod (3)=2$. Sometimes we have $q=\left\lfloor\frac{n}{3}\right\rfloor$ and $s=\left\lceil\frac{2 n}{3}\right\rceil$ and at other times, we have $q=\left\lceil\frac{n}{3}\right\rceil$ and $s=\left\lfloor\frac{2 n}{3}\right\rfloor$. We decided to examine the two cases interactively on AGX for every $n$ up to 24, and we observed that the objective function has the same value in both cases $\left(q=\left\lfloor\frac{n}{3}\right\rfloor\right.$ or $\left.q=\left\lceil\frac{n}{3}\right\rceil\right)$.

AGX did not find any conjecture on the relation between the objective function $\lambda_{1}(G)+\lambda_{1}(\bar{G})$ and the order when using all the presumably extremal graphs obtained by AGX. But when we separated the set of graphs into three subsets, with $n \bmod (3)=0$ for the first subset, $n \bmod (3)=1$ for the second one and $n \bmod (3)=2$ for the third one, AGX did not find anything about the two first subsets but suggested the following linear relation for the third one $(n \bmod (3)=2)$

$$
\lambda_{1}(G)+\lambda_{1}(\bar{G})=\frac{4}{3} n-\frac{5}{3} .
$$

The difficulty in proving Conjectures 3.1 and 4.1 is that we have almost no lemmas on the behavior of the corresponding invariant under local graph transformations. 
Experiments with GRAPH (Cvetković and Simić 2005), newGRAPH (Stevanović D, http://www.mi.sanu.ac.yu/newgraph/) and AGX would be useful in producing conjectures for such lemmas (e.g. adding an edge, rotating an edge etc.). However, for the irregularity of a graph (involved in Conjecture 2.1) we do have such lemmas.

Conjecture 4.1 was proved in Aouchiche et al. (2008a) in some particular cases.

Proposition 4.2 Let $G$ be a connected graph on $n$ vertices with minimum degree $\delta$ and maximum degree $\Delta$.

- If $\Delta-\delta \leq \frac{n-2}{3}$, then

$$
\lambda_{1}(G)+\lambda_{1}(\bar{G}) \leq \frac{4}{3} n-\frac{5}{3} .
$$

- If $G$ is a complete split graph $\mathrm{CS}(n, q)$, then

$$
\lambda_{1}(G)+\lambda_{1}(\bar{G}) \leq \frac{4}{3} n-\frac{5}{3}- \begin{cases}\frac{3 n-2-\sqrt{9 n^{2}-12 n+12}}{6} & \text { if } n \bmod (3)=1 \\ 0 & \text { if } n \bmod (3)=2 \\ \frac{3 n-1-\sqrt{9 n^{2}-6 n+9}}{6} & \text { if } n \bmod (3)=0\end{cases}
$$

with equality if and only if $q=\left\lfloor\frac{n}{3}\right\rfloor$ for all $n$ or $q=\left\lceil\frac{n}{3}\right\rceil$ for $n \bmod (3)=2$.

The first point of the above proposition was proved using the well-known inequality $\lambda_{1}(G) \leq \Delta(G)$ (see e.g. Cvetković et al. 1995) and the fact that $\Delta(\bar{G})=n-1-\delta(G)$. The second point was proved by using a combination of the following inequalities proved by Stanley (1987) and Hong et al. (2001), respectively:

$$
\begin{aligned}
\text { Stanley (1987) } & \lambda_{1} \leq \frac{-1+\sqrt{1+8 m}}{2} \\
\text { Hong et al. (2001) } & \lambda_{1} \leq \frac{\delta-1+\sqrt{(\delta+1)^{2}+4(2 m-\delta n)}}{2} .
\end{aligned}
$$

\section{Energy of a graph}

The energy $E(G)$ of a graph $G$, introduced by Gutman (1978) in 1978 (see Gutman 2001 for a survey), is defined as the sum of the absolute values of its eigenvalues, i.e.

$$
E(G)=\sum_{i=1}^{n}\left|\lambda_{i}(G)\right|=2 \sum_{\lambda_{i}>0} \lambda_{i}(G)=2 \sum_{\lambda_{i}<0}\left|\lambda_{i}(G)\right| .
$$

A lollipop $\operatorname{Lol}_{n, g}$, with $n \geq g \geq 3$, is a graph obtained from a cycle $C_{g}$ and a path $P_{n-g}$ by adding an edge between a vertex from the cycle and an endpoint from the path (see Fig. 3 for $\mathrm{Lol}_{10,6}$ ). $\mathrm{Lol}_{n, n-1}$ is called the short lollipop while $\mathrm{Lol}_{n, 3}$ is the long lollipop and $\mathrm{Lol}_{n, n}$ is the cycle $C_{n}$.

In order to find lower and upper bounds on the energy, Caporossi et al. (1999a) used the AGX system. They found the following conjectures afterwards proved by hand. 
Fig. 3 The lollipop $\mathrm{Lol}_{10,6}$

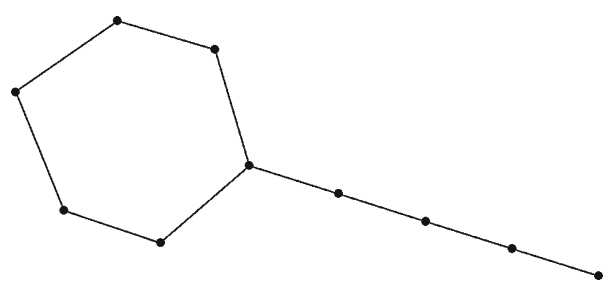

Theorem 5.1 Let $G$ be a simple graph on $n$ vertices and $m$ edges with energy $E$. Then

1. $E \geq 4 m / n$;

2. $E \geq 2 \sqrt{m}$ with equality if and only if $G$ is a complete bipartite graph plus possibly some isolated vertices;

3. if $G$ is connected, $E \geq 2 \sqrt{n-1}$ with equality if and only if $G$ is the star $S_{n}$;

4. $E \leq 2 m$ with equality if and only if $G$ is composed of disjoint edges and possibly isolated vertices.

In this study, the particular case of unicyclic graphs was considered. Some unicyclic graphs that maximize the energy are given in Fig. 4. The following conjecture was stated.

Conjecture 5.2 Among unicyclic graphs on $n$ vertices the cycle $C_{n}$ has maximal energy if $n \leq 7$ and $n=9,10,11,13$ and 15. For all other values of $n$ the unicyclic graph with maximum energy is the lollipop $\operatorname{Lol}_{n, 6}$.

A weaker form of this conjecture was proved by Hou et al. (2002) in the following theorem.

Theorem 5.3 Let $G$ be a connected, unicyclic and bipartite graph on $n \geq 7$ vertices and $G ¥ C_{n}$. Then $E(G) \leq E\left(\operatorname{Lol}_{n, 6}\right)$.

A few years later, Huo et al. (2011) and Andriantiana (2011) proved independently the following result.

Theorem 5.4 For $n=8,12,14$ and $n \geq 16$, we have $E\left(\operatorname{Lol}_{n, 6}\right)>E\left(C_{n}\right)$.

Again, in another paper, Huo et al. (2011) solved completely the problem to get
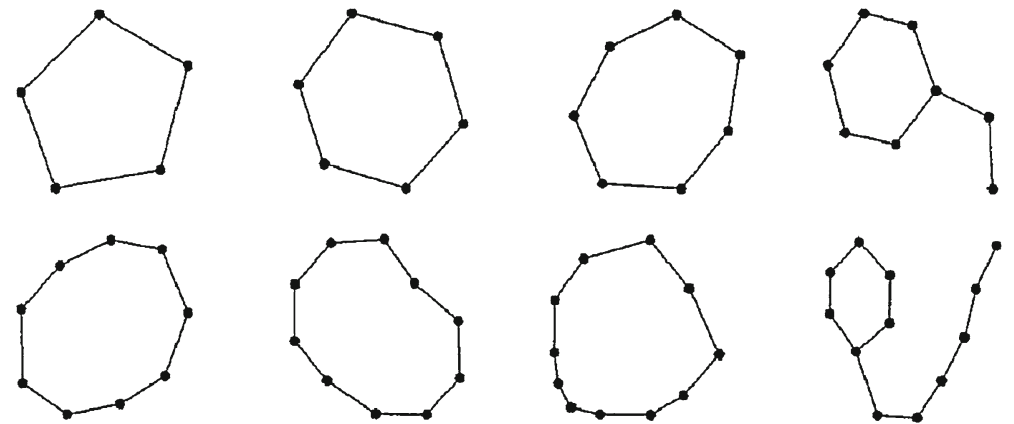

Fig. 4 Unicyclic graphs with largest energy for $n=5-12$ 
Fig. 5 The graph $D_{n}$

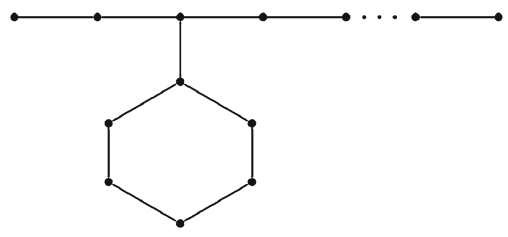

Fig. 6 The $P_{n}^{6 ; 6}$ graph

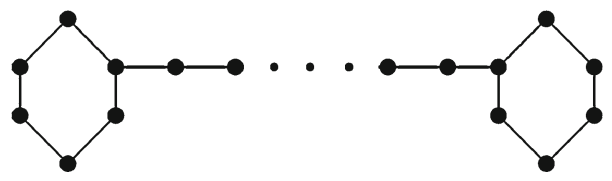

Theorem 5.5 Among all unicyclic graphs on $n$ vertices, the cycle $C_{n}$ has maximal energy if $n \leq 7$ but $n \neq 4$, and $n=9,10,11,13$ and $15 ;$ Lol $_{4,3}$ has maximal energy if $n=4$. For all other values of $n$, the unicyclic graph with maximal energy is $\operatorname{Lol}_{n, 6}$.

To prove their theorem, Huo et al. (2011) used the Coulson integral formula (Coulson 1940) and proved several lemmas.

The extremal graph in the above theorem are bipartite. The case of non-bipartite graphs was studied by Andriantiana and Wagner (2011) who gave the following theorem.

Theorem 5.6 Among all non-bipartite unicyclic graphs in $U_{n}(n \geq 3), \operatorname{Lol}_{n, 3}$ has maximum energy if $n$ is even, and $C_{n}$ has maximum energy if $n$ is odd.

Andriantiana and Wagner (2011) solved a conjecture posed by Gutman et al. (2007) about connected unicyclic graphs with the second largest energy.

Theorem 5.7 Let $D_{n}$ be the graph obtained by joining a vertex of the cycle $C_{6}$ and the third vertex in a path $P_{n-6}$ by an edge (see Fig. 5). For $n \geq 28, D_{n}$ is the unicyclic graph with second-largest energy.

The case of bipartite graphs was solved by Hua (2007).

Theorem 5.8 Let $G \in U_{n} \backslash\left\{\operatorname{Lol}_{n, k} \mid k=3,4, \ldots n\right\}$ be a bipartite unicyclic graph. For $n \geq 13$, if $G \neq D_{n}$, we have $E(G)<E\left(D_{n}\right)$.

The problem of finding bicyclic graphs with maximum energy was also widely studied. It was posed by Gutman and Vidović (2001). Among others, they conjectured the following. Before the statement of the conjecture, recall that a molecular graph is a connected graph with maximum degree at most 4.

Conjecture 5.9 For $n=14$ and $n \geq 16$, the bicyclic molecular graph of order $n$ with maximal energy is the molecular graph of the $\alpha, \beta$ diphenyl-polyene $\mathrm{C}_{6} \mathrm{H}_{5}(\mathrm{CH})_{n-12} \mathrm{C}_{6} \mathrm{H}_{5}$, which is represented by the graph $P_{n}^{6 ; 6}$ obtained from two copies of the cycle $C_{6}$ and a path $P_{n-12}$ by attaching with an edge each endpoint of the path to on cycle (see Fig. 6).

Furtula et al. (2008) showed by numerical computations that the conjecture is true up to $n=50$. Li and Zhang (2007) gave a partial solution to the conjecture in the case 

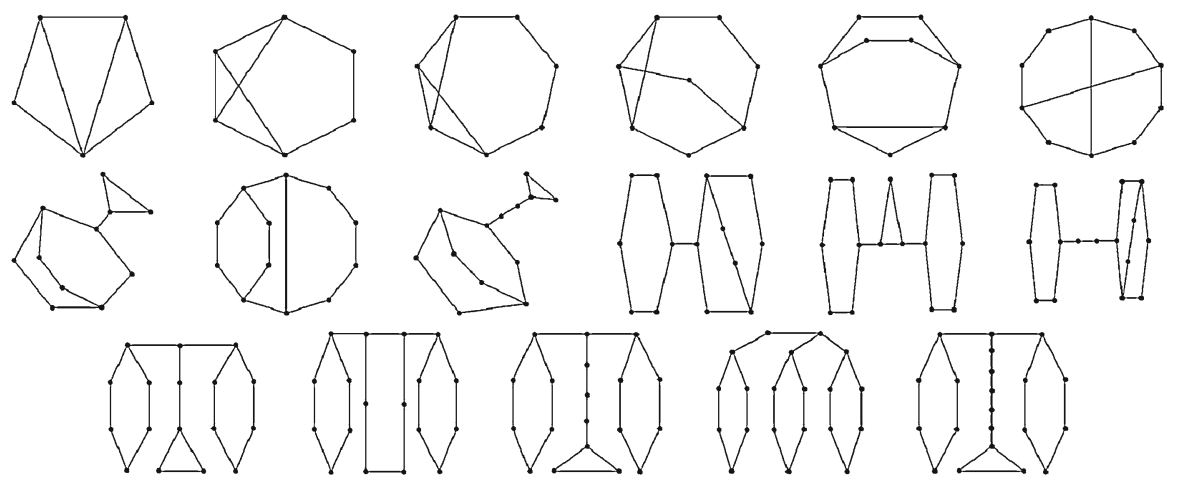

Fig. 7 Tricyclic graphs that presumably maximize energy for $n=6, \ldots, 21$
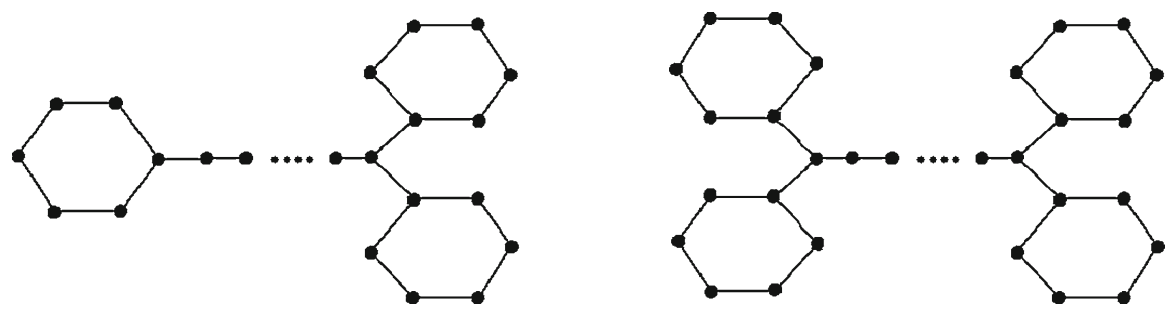

Fig. 8 The tricyclic $P_{n}^{6,6 ; 6}$ and the quadricyclic $P_{n}^{6,6 ; 6,6}$ graphs

of bipartite bicyclic graphs. The complete solution, in that case, was given by Huo et al. (2011).

Theorem 5.10 Let $G$ be any connected, bipartite bicyclic graph on $n \geq 12$ vertices. Then $E(G) \leq E\left(P_{n}^{6 ; 6}\right)$ with equality if and only if $G \equiv P_{n}^{6 ; 6}$.

The problem of finding the tricyclic graphs maximizing the energy remains open. Experiments using AutoGraphiX led us to conjecture that for $n=6, \ldots, 21$, the energy is maximum for the graphs given in Fig. 7 .

For $n \geq 22$, we have a general conjecture obtained with AutoGraphiX. First let $P_{n}^{6,6 ; 6}$ denote the graph on $n \geq 20$ obtained from three copies of $C_{6}$ and a path $P_{n-18}$ by adding a single edge between each of two copies of $C_{6}$ to one endpoint of the path and a single edge from the third $C_{6}$ to the other endpoint of the $P_{n-18}$. The graph $P_{n}^{6,6 ; 6}$ is illustrated in Fig. 8 (left).

Conjecture 5.11 Let $G$ be a tricyclic graph on $n$ vertices with $n=20$ or $n \geq 22$. Then $E(G) \leq E\left(P_{n}^{6,6 ; 6}\right)$ with equality if and only if $G \equiv P_{n}^{6,6 ; 6}$.

The problem of finding the quadricyclic (also called tetracyclic) graphs maximizing the energy remains open. Experiments using AutoGraphiX led us to conjecture that for $n=5, \ldots, 27$, the energy is maximum for the graphs given in Fig. 9 .

For $n \geq 26$, we have a general conjecture obtained with AutoGraphiX. First, let $P_{n}^{6,6 ; 6,6}$ denote the graph on $n \geq 26$ obtained from four copies of $C_{6}$ and a path $P_{n-24}$ by adding a single edge between each of two copies of $C_{6}$ to one endpoint of the path 

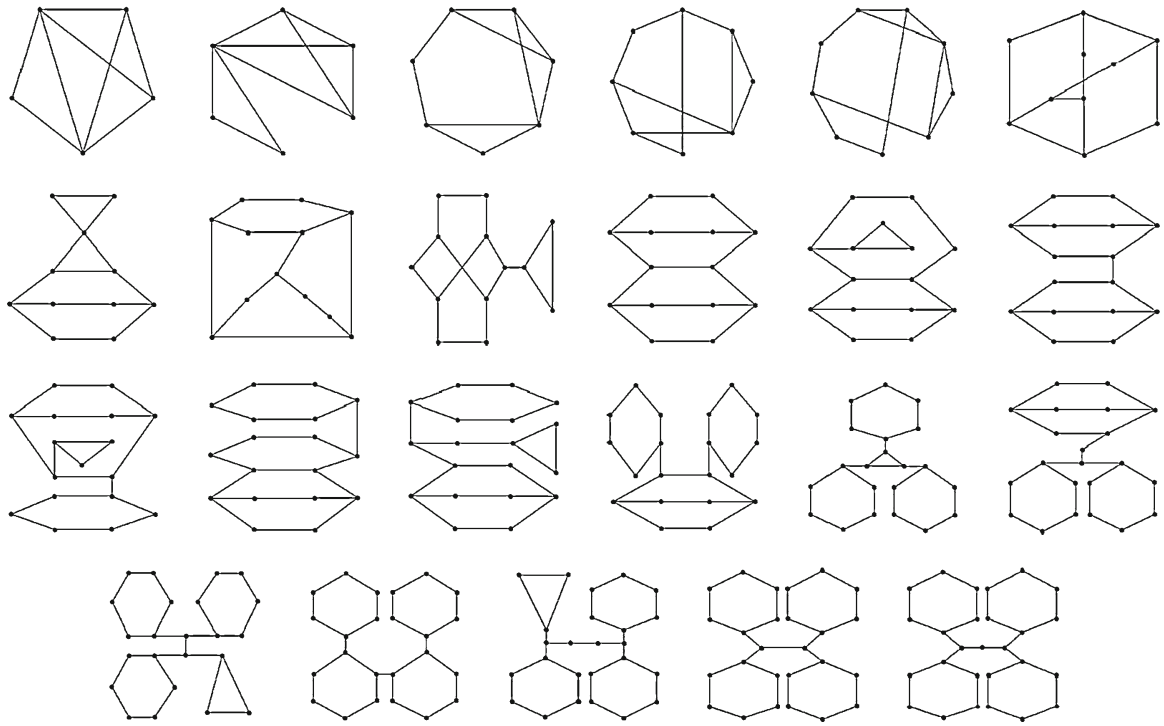

Fig. 9 Quadricyclic graphs that presumably maximize energy for $n=6, \ldots, 21$

and a single edge from each of the two other copies of $C_{6}$ to the other endpoint of the $P_{n-24}$. The graph $P_{n}^{6,6 ; 6,6}$ is illustrated in Fig. 8 (right).

Conjecture 5.12 Let $G$ be a tetracyclic graph on $n$ vertices with $n \geq 26$. Then $E(G) \leq E\left(P_{n}^{6,6 ; 6,6}\right)$ with equality if and only if $G \equiv P_{n}^{6,6 ; 6,6}$.

In view of Theorem 5.10, Conjectures 5.11 and 5.12, we are able to state a more general conjecture about the structure of the graphs maximizing the energy. Let $p, q, r$ be integers such that $r=p+q$. For an integer $n$ such that $n \geq 6 r+2$, let $P_{n}^{p \times 6 ; q \times 6}$ be the graph obtained from $r$ copies of $C_{6}$ and a path $P_{n-6} r$ with endpoints $u$ and $v$, by adding an edge between $u$ and each of $p$ copies of $C_{6}$ and an edge between $v$ and each of the $q$ other copies of $C_{6}$. It is evident that $P_{n}^{6 ; 6} \equiv P_{n}^{1 \times 6 ; 1 \times 6}, P_{n}^{6,6 ; 6} \equiv P_{n}^{2 \times 6 ; 1 \times 6}$ and $P_{n}^{6,6 ; 6,6} \equiv P_{n}^{2 \times 6 ; 2 \times 6}$. Now the general conjecture is the following.

Conjecture 5.13 Let $r$ and $n$ be positive integers such that $n \geq 6 r+4$. Then $E(G) \leq$ $E\left(P_{n}^{p \times 6 ; q \times 6}\right)$, where $p=\lfloor r / 2\rceil$ and $q=\lfloor r / 2\rfloor$, with equality if and only if $G \equiv$ $P_{n}^{p \times 6 ; q \times 6}$.

\section{References}

Alberston MO (1997) The irregularity of a graph. Ars Combin 46:215-225

Amin AT, Hakimi SL (1972) Upper bounds on the order of a clique of a graph. SIAM J Appl Math 22: 569-573

Andriantiana EOD (2011) Unicyclic bipartite graphs with maximum energy. MATCH Commun Math Comput Chem 66:913-926

Andriantiana EOD, Wagner S (2011) Unicyclic graphs with large energy. Linear Algebra Appl 435: 1399-1414 
Aouchiche M (2006) Comparaison automatisée d'invariants en théorie des graphes. PhD Thesis (French), École Polytechnique de Montréal

Aouchiche M, Hansen P (2005) Recherche á voisinage variable de graphes extémaux. XIII. Á propos de la maille. (French) [Variable neighborhood search for extremal graphs. XIII. Girth]. RAIRO Oper Res 39:275-293

Aouchiche M, Hansen P (2007a) On a conjecture about the Randić index. Discrete Math 307:262-265

Aouchiche M, Hansen P (2007b) Automated results and conjectures on average distance in graphs. In: Graph theory in Paris. Trends in mathematics. Birkhäuser, Basel, pp 21-36

Aouchiche M, Hansen P (2009) Bounding average distance using order and minimum degree. Graph Theory Notes N Y 56:21-29

Aouchiche M, Hansen P (2010) On a conjecture about the Szeged index. Eur J Combin 31:1662-666

Aouchiche M, Hansen P (2011) Proximity and remoteness in graphs: results and conjectures. Networks 58:95-102

Aouchiche M, Hansen P (2012) A survey of Nordhaus-Gaddum type relations. Discrete Appl Math (in press) (available online 16 January 2012)

Aouchiche M, Caporossi G, Hansen P (2001) Variable neighborhood search for extremal graphs 8. variations on graffiti 105. Congr Numer 148:129-144

Aouchiche M, Bonnefoy J-M, Fidahoussen A, Caporossi G, Hansen P, Hiesse L, Lacheré J, Monhait A (2005) Variable neighborhood search for extremal graphs. 14. The AutoGraphiX 2 System. In: Liberti L, Maculan N (eds) Global optimization: from theory to implementation. Springer, Berlin, pp 281-310

Aouchiche M, Hansen P, Zheng M (2006) Variable neighborhood search for extremal graphs. XVIII. Conjectures and results about the Randić index. MATCH Commun Math Comput Chem 56:541-550

Aouchiche M, Caporossi G, Hansen P (2007a) Variable neighborhood search for extremal graphs. 20. Automated comparison of graph invariants. MATCH Commun Math Comput Chem 58:365-384

Aouchiche M, Hansen P, Zheng M (2007b) Variable neighborhood search for extremal graphs. XIX. Further conjectures and results about the Randić index. MATCH Commun Math Comput Chem 58:83-102

Aouchiche M, Bell FK, Cvetković D, Hansen P, Rowlinson P, Simic S, Stevanović D (2008a) Variable neighborhood search for extremal graphs. 16. Some conjectures related to the largest eigenvalue of a graph. Eur J Oper Res 191:661-676

Aouchiche M, Brinkmann G, Hansen P (2008b) Variable neighborhood search for extremal graphs. XXI. Conjectures and results about the independence number. Discrete Appl Math 156:2530-2542

Aouchiche M, Favaron O, Hansen P (2009a) Recherche á voisinage variable de graphes extrémaux. XXVI. Nouveaux résultats sur la maille. (French) [Variable neighborhood search for extremal graphs. XXVI. New results for the girth]. RAIRO Oper Res 43:339-358

Aouchiche M, Favaron O, Hansen P (2009b) Variable neighborhood search for extremal graphs. XXII. Extending bounds for independence to upper irredundance. Discrete Appl Math 157:3497-3510

Aouchiche M, Hansen P, Stevanović D (2009) Variable Neighborhood Search for Extremal Graphs. XVII. Further conjectures and results about the index. Discuss Math Graph Theory 29:15-37

Aouchiche M, Hansen P, Stevanović D (2010) A sharp upper bound on algebraic connectivity using domination number. Linear Algebra Appl 432:2879-2893

Aouchiche M, Hansen P, Lucas C (2011) On the extremal values of the second largest $Q$-eigenvalue. Linear Algebra Appl 435:2591-2606

Belhaiza S, Abreu NMM, Hansen P, Oliveira CS (2005) Variable neighborhood search for extremal graphs 11. bounds on algebraic connectivity. In: Avis D, Hertz A, Marcotte O (eds) Graph theory and combinatorial optimization, GERAD 25th anniversary series, vol 8. Springer, New York, pp 1-16

Bell FK (1992) A note on the irregularity of graphs. Linear Algebra Appl 161:45-54

Caporossi G (2000) Découverte par Ordinateur en Théorie de Graphes (French) PhD Thesis. École Polytechnique de Montréal

Caporossi G, Cvetković D, Gutman I, Hansen P (1999a) Variable neighborhood search for extremal graphs 2. Finding graphs with extremal energy. J Chem Inf Comput Sci 39:984-996

Caporossi G, Gutman I, Hansen P (1999b) Variable neighborhood search for extremal graphs 4. Chemical trees with extremal connectivity index. Comput Chem 23:469-477

Caporossi G, Gutman I, Hansen P, Pavlović L (2003) Graphs with maximum connectivity index. Computat Biol Chem 27:85-90

Caporossi G, Hansen P (2000) Variable Neighborhood Search for Extremal Graphs: I. The AutoGraphiX System. Discrete Math 212:29-44 
Caporossi G, Hansen P (2004) Variable neighborhood search for extremal graphs: V. Three ways to automate finding conjectures. Discrete Math 276:81-94

Collatz L, Sinogowitz U (1957) Spektren endlicher Grafen. Abh Math Sem Univ Hamburg 21:63-77

Coulson CA (1940) On the calculation of the energy in unsaturated hydrocarbon molecules. Proc Cambridge Phil Soc 36:201-203

Csikvári P (2009) On a conjecture of V. Nikiforov. Discrete Math 309:4522-4526

Cvetković D, Doob M, Sachs H (1995) Spectra of graphs- theory and applications, 3rd edn. Johann Ambrosius Barth Verlag, Heidelberg

Cvetković D, Simić S (2005) Graph theoretical results obtained by the support of the expert system "GRAPH"—-an extended survey. In: Graphs and discovery. DIMACS Ser Discrete Math Theoret Comput Sci 69:39-70

Cvetković D, Simić S, Caporossi G, Hansen P (2001) Variable neighborhood search for extremalpagination graphs. III. On the largest eigenvalue of color-constrained trees. Linear Multilinear Algebra 49:pagination 143-160

di Battista G, Eades P, Tamassia R, Tollis IG (1994) Algorithms for drawing graphs: an annotated bibliography. Comput Geom Theory Appl 4(5):235-282

di Battista G, Eades P, Tamassia R, Tollis IG (1999) Graph drawing: Algorithms for the visualization of graphs. Prentice Hall, Englewood Cliffs

Fajtlowicz S, Fowler P, Hansen P, Janowitz M, Roberts f (eds) (2005) Graphs and discovery. DIMACS Ser Discrete Math Theor Comput Sci, vol 69. AMS, New York

Finck H-J (1968) On the chromatic numbers of a graph and its complement, Theory of Graphs (Proc. Colloq., Tihany, 1966). Academic Press, New York, pp 99-113

Fowler PW, Hansen P, Caporossi G, Soncini A (2001) Variable neighborhood search for extremal graph 7. Polyenes with maximum homo-lumo gap. Chem Phys Lett 342:105-112

Furtula B, Radenković S, Gutman I (2008) Bicyclic molecular graphs with the greatest energy. J Serb Chem Soc 73:431-433

Gregory AD, Hershkovitz D, Kirkland SJ (2001) The spread of the spectrum of a graph. Linear Algebra Appl 332-334:23-35

Gutman I (1978) The energy of a graph. Ber Math Statist Sekt Forsch-ungsz Graz 103:1-22

Gutman I (2001) The energy of a graph: Old and new results. In: Betten A, Kohnert A, Laue R, Wassermann A (eds) Algebraic combinatorics and applications. Springer, Berlin, pp 196-211

Gutman I, Furtula B, Hua H (2007) Bipartite unicyclic graphs with maximal, second-maximal, and thirdmaximal energy. Match 58:75-82

Gutman I, Hansen P, Mélot H (2005) Variable neighborhood search for extremal graphs. 10. Comparison of irregularity indices for chemical trees. J Chem Inf Model 45:222-230

Gutman I, Miljković O, Caporossi G, Hansen P (1999) Alkanes with small and large randić connectivity indices. Chem Phys Lett 306:366-372

Gutman I, Vidović D (2001) Quest for molecular graphs with maximal energy: a computer experiment. J Chem Inf Sci 41:1002-1005

Hansen P (2005) How far is, should and could be conjecture-making in graph theory an automated process? In: Fajtlowicz S, Fowler P, Hansen P, Janowitz M, Roberts f (eds) Graphs and discovery. DIMACS Ser Discrete Math Theor Comput Sci, vol 69. AMS, New York, pp 189-229

Hansen P, Mélot H (2003) Variable neighborhood search for extremal graphs 6. Analyzing bounds for the connectivity index. J Chem Inf Comp Sci 43:1-14

Hansen P, Mélot H (2005) Variable neighborhood search for extremal graphs 9. Bounding the irregularity of a graph. In: Fajtlowicz S, Fowler P, Hansen P, Janowitz M, Roberts f (eds) Graphs and discovery. DIMACS Ser Discrete Math Theor Comput Sci, vol 69. AMS, New York

Hansen P, Mladenović N (2001) Variable neighborhood search: principles and applications. Eur J Oper Res 130:449-467

Hansen P, Mélot H, Gutman I (2005) Variable neighborhood search for extremal graphs 12. A note on the variance of bounded degrees in graphs. MATCH Commun Math Comput Chem 54:221-232

Hong Y (1988) A bound on the spectral radius of graphs. Linear Algebra Appl 108:135-139

Hong Y, Shu J-L (2000) A sharp upper bound for the spectral radius of the Nordhaus-Gaddum type. Discrete Math 211:229-232

Hong Y, Shu J-L, Fang K (2001) A sharp upper bound of the spectral radius of graphs. J Combin Theory Ser B 81(2):177-183

Hou Y, Gutman I, Woo C-W (2002) Unicyclic graphs with maximal energy. Linear Algebra Appl 356:27-36 
Hua H (2007) Bipartite unicyclic graphs with large energy. MATCH Commun Math Comput Chem 58: $57-73$

Huo B, Ji S, Li X, Shi Y (2011) Solution to a conjecture on the maximal energy of bipartite bicyclic graphs. Linear Algebra Appl 435:804-810

Huo B, Li X, Shi Y (2011) Complete solution to a problem on the maximal energy of unicyclic bipartite graphs. Linear Algebra Appl 434:1370-1377

Huo B, Li X, Shi Y (2011) Complete solution to a conjecture on the maximal energy of unicyclic graphs. Eur J Combin 32:662-673

Knuth D (1993) The Stanford graphbase: a platform for combinatorial computing. Addison-Wesley, Reading

Li X (1996) The relations between the spectral radius of the graphs and their complement (Chinese). J North China Technol Inst 17:297-299

Li X, Zhang J (2007) On bicyclic graphs with maximal energy. Linear Algebra Appl 427:87-98

Mehlhorn K, Nähger S (1995) Leda: a platform for combinatorial and geometric computing. Commun ACM 38(1):96-102

Mladenović N, Hansen P (1997) Variable neighborhood search. Comput Oper Res 24:1097-1100

Nikiforov V (2002) Some inequalities for the largest eigenvalue of a graph. Combin Probab Comput 11: $179-189$

Nikiforov V (2007) Eigenvalue problems of Nordhaus-Gaddum type. Discrete Math 307:774-780

Nordhaus EA, Gaddum JW (1956) On complementary graphs. Am Math Monthly 63:175-177

Nosal E (1970) Eigenvalues of graphs. University of Calgary, Master Thesis

Petrović M (1983) On graphs whose spectral spread does not exceed 4. Publ Inst Math (Beograd) 34(48):169-174

Pisanski T, Zitnik A (2002) Interactive conjecturing with vega. In: Fajtlowicz S, Fowler P, Hansen P, Janowitz M, Roberts f (eds) Graphs and discovery. DIMACS Ser Discrete Math Theor Comput Sci, vol 69. AMS, New York

Sedlar J, Vukičević D, Aouchiche M, Hansen P (2008) Variable neighborhood search for extremal graphs. XXV. Products of connectivity and distance measures. Graph Theory Notes New York 55:6-13

Shi L (2007) Bounds on the (Laplacian) spectral radius of graphs. Linear Algebra Appl 422:755-770

Shu J-L, Wu Y (2003) The spread of the unicyclic graphs. electronic file available at http://www.math.ecnu. edu.cn/preprint/2003-004.pdf

Skiena S (1990) Implementing discrete mathematics: combinatorics and graph theory with mathematica. Addison-Wesley, Menlo Park

Stanley RP (1987) A bound on the spectral radius of graphs with $e$ edges. Linear Algebra Appl 87:267-269

Stevanović D, Aouchiche M, Hansen P (2008) On the spectral radius of graphs with a given domination number. Linear Algebra Appl 428:1854-1864 